\title{
Formal Model Reduction
}

\author{
Jérôme Feret \\ Laboratoire d'informatique de l'École normale supérieure \\ (INRIA/ÉNS/CNRS) \\ www.di.ens.fr/ feret
}

Modelers of molecular signaling networks must cope with the combinatorial explosion of protein states generated by post-translational modifications and complex formations. Rule-based models provide a powerful alternative to approaches that require an explicit enumeration of all possible molecular species of a system [12]. Such models consist of formal rules stipulating the (partial) contexts for specific protein-protein interactions to occur. The behavior of the models can be formally described by stochastic or differential semantics. Yet, the naive computation of these semantics does not scale to large systems, because it does not exploit the lower resolution at which rules specify interactions.

We present a formal framework for constructing coarse-grained systems. We instantiate this framework with two abstract domains. The first one tracks the flow of information between the different regions of chemical species, so as to detect and abstract away some useless correlations between the state of sites of molecular species. The second one detects pairs of sites having the same capabilities of interactions, and abstract away any distinction between them.

The result of our abstraction is a set of molecular patterns, called fragments, and a system which describes exactly the concentration (or population) evolution of these fragments. The method never requires the execution of the concrete rule-based model and the soundness of the approach is described and proved by abstract interpretation [3]. Unlike our previous analysis [4, our fragments are heterogeneous. The cutting of a protein into portions may depend on its position within the molecular species. This matches more closely with the flow of information. Indeed, within a molecular species, the behavior of a protein may be driven by the state of a site without being driven by the state of the same site in other instances of the protein. Our new analysis exploits this efficiently.

(Joint work with F. Camporesi, V. Danos, W. Fontana, R. Harmer, and J. Krivine.)

\section{References}

1. Danos, V., Laneve, C.: Formal molecular biology. TCS 325(1) (2004)

2. Blinov, M.L., Faeder, J.R., Hlavacek, W.S.: BioNetGen: software for rule-based modeling of signal transduction based on the interactions of molecular domains. Bioinformatics 20 (2004)

3. Cousot, P., Cousot, R.: Abstract interpretation: a unified lattice model for static analysis of programs by construction or approximation of fixpoints. In: POPL (1977)

4. Feret, J., Danos, V., Krivine, J., Harmer, R., Fontana, W.: Internal coarse-graining of molecular systems. PNAS (2009) 